\title{
PERFORMANCE OF THE BEAM STABILIZING FEEDBACK SYSTEMS AT CESR*
}

\author{
M. Billing ${ }^{\dagger}$, G. Codner, D. Hartill, R. Meller, J. Sikora, V. Vescherevich, \\ Laboratory of Nuclear Studies, Cornell University, Ithaca, NY 14853, USA
}

\begin{abstract}
The operation of CESR as an electron-positron collider using trains of bunches requires the use of beam stabilizing feedback systems at routine operating currents. These systems operate on the longitudinal and both transverse modes of dipole oscillation. The experience in routine operations and feedback system performance will be presented.
\end{abstract}

\section{INTRODUCTION}

Transverse and longitudinal feedback are required for CESR operation. Horizontally, there is a strong instability due to trapped electrons near the distributed ion pumps in the ring [1]. The instability strength peaks at about $80 \mathrm{~mA}$ of total positron current, and is largely independent of the number of bunches. With large numbers of bunches, the lower per bunch current requires increased signal gain from the electronics. Vertical feedback is active, but is not essential for operation.

Single beams of electrons or positrons will become longitudinally unstable at about $140 \mathrm{~mA}$. The two-beam instability threshold is higher at $200 \mathrm{~mA}$. When unstable, the longitudinal motion limits at a fairly low value, but this is enough to produce a significant $(\sim 20 \%)$ reduction in luminosity for colliding beam conditions. With feedback in operation the beam routinely remains stable at total currents above $700 \mathrm{~mA}$, a factor of three higher than the longitudinal instability threshold. However, there have been some instances of loss of stability during injection, during transition to collisions and during collisions without any apparent transitions. Generally, once the beam becomes unstable, current must be reduced substantially in order to regain stability since the feedback system does not have sufficient power to stabilize a beam when there is significant longitudinal motion.

Operational concerns identified so far have been: 1) longitudinal instabilities induced by electrostatic separator arcs, which produce a sudden (within a single turn) change in beam trajectory and a resulting partial beam loss. The effect on the longitudinal feedback is a large signal transient. Presumably, the beam goes unstable during the recovery from the transient. 2) Low frequency signals modulating the CESR RF cavities producing a beam response at a level high enough to saturate the feedback power amplifier, and 3) occasional failures in which the hardware enters a dysfunctional state. Reinitializing the hardware restores normal operation.

\footnotetext{
*Work supported by the National Science Foundation

†mgb@a.cesr10.lns.cornell.edu
}

\section{SYSTEM DESCRIPTION}

CESR multiple bunch feedback has recently been upgraded with the digital signal processor (DSP) using a direct sampling ADC (analog to digital converter) that operates at $71.4 \mathrm{MHz}$, capable of processing the 14 nanosecond spaced bunches in a single channel [2]. The single channel approach eliminates the need for signal stretchers and demultiplexed channels for each bunch.

The DSPs, which are used for all six CESR feedback subsystems (horizontal, vertical and longitudinal for electrons and positrons), now include more flexible programmable filters, larger diagnostic buffers and programmable diagnostic buffer triggers. Plug-in daughterboards provide for modular functions.

All of the required differences between functions are software programmable. The same DSP's are also used for feedback in the CESR synchrotron with a firmware change to account for the difference in the number of RF buckets [3].

\subsection{Transverse Feedback}

The transverse feedback systems uses pairs of combined beam buttons to develop position sensitive signals that are then applied to a hybrid combiner that provide sum and difference signals. The difference signal is used for feedback. The vertical system has an additional front end board that accepts the vertical sum signal for measuring bunch current. The DSP supplies an error signal to the modulator, which generates a waveform suitable as a source for the 200 watt amplifier which drives the stripline kicker. This waveform is now a 7 nanosecond pulse to allow injection of bunches spaced 6 and 8 nanoseconds apart, an ongoing development project. At $3.5 \mathrm{~ns}$, the electrical length of the kicker will accommodate the more closely-spaced bunches.

\subsection{Longitudinal Feedback}

Whereas CESR longitudinal feedback previously used the horizontal stripline kicker, it now uses a cavity kicker. The cavity is driven by a 200 watt traveling wave tube (TWT) amplifier. The kicker was adapted from the DAФNE design [4]. Separation of these systems has simplified feedback operation significantly in addition to providing more dynamic range.

In the longitudinal feedback system, four beam button signals are combined using microstrip cross-combiners with excellent wideband impedance matching for very low interbunch crosstalk and small transverse position dependence. This intensity signal is applied to a phase 

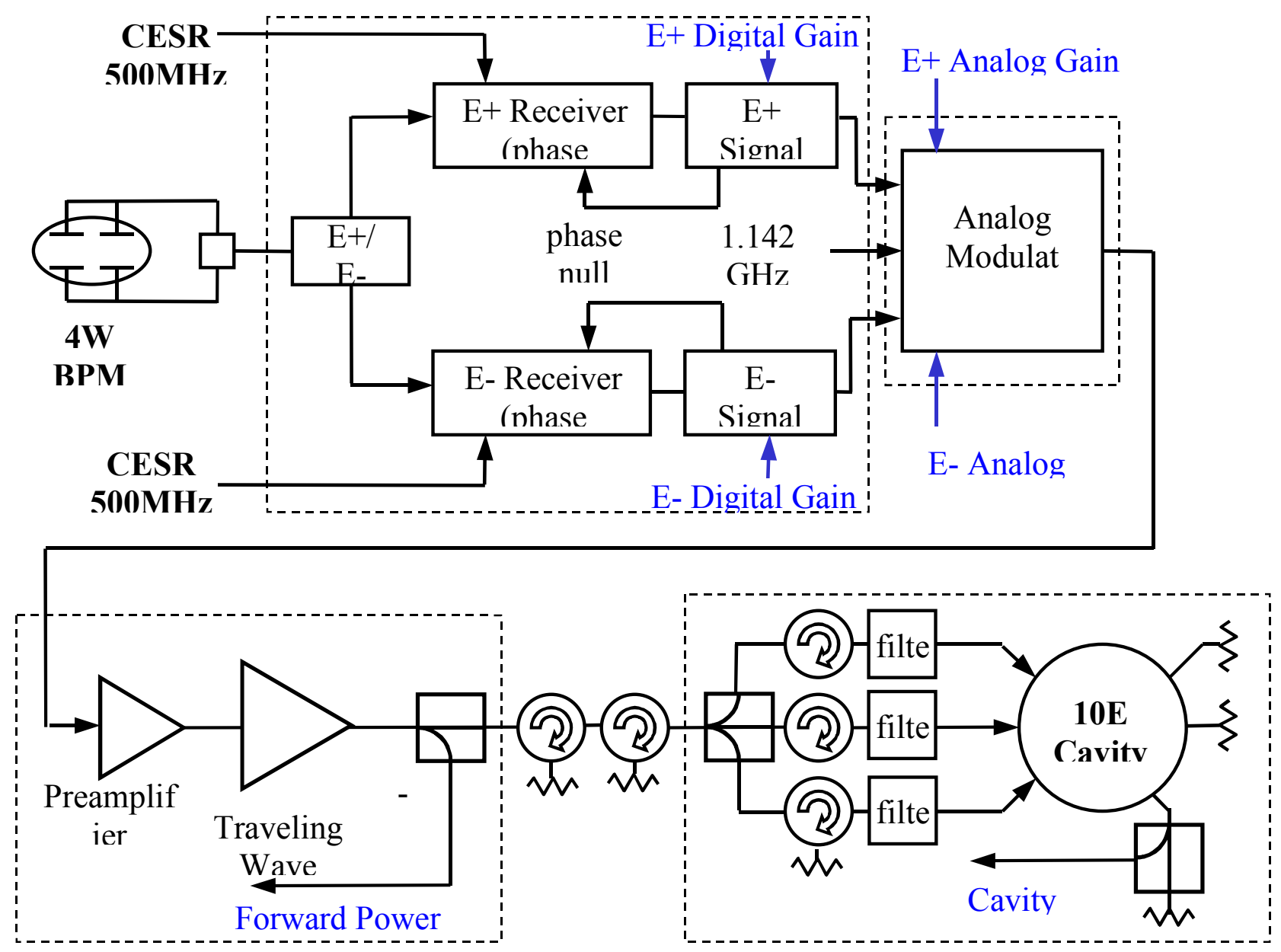

Figure 1: CESR longitudinal feedback block diagram.

detector with the CESR $500 \mathrm{MHz}$ as a reference, producing a signal with sensitivity of 0.16 radians per volt. The detected signal is connected to the DSP.

The digitized error signal is applied to the pedestal circuit which does an analog subtraction of each averaged bunch signal from each incoming bunch signal. Pedestal cancellation is vital for achieving the gain necessary to maintain longitudinal stability at the highest currents. The filtered pedestal signal is also used in a closed loop to maintain quadrature of the phase detector in the receiver. In the transverse feedback system, the pedestal signal for the sum signal represents the bunch current.

In the main feedback path, the error signal is applied to digital lowpass and bandpass infinite impulse response (IIR) filters. The bandpass filter is critical for reducing the noise level as CESR longitudinal feedback gain is limited by noise in the $500 \mathrm{MHz}$ local oscillator.

The filtered error signal is applied to an output FIFO for rough control of phase and is converted to an analog signal. Because the signal is at the second-order lowpass IIR filter cutoff frequency, the necessary 90 degree phase shift between position detection and energy kick is achieved with zero turns delay. The bandpass filter contributes roughly zero phase because it is set to the synchrotron tune. The Q of this filter is 6 , which means that it has rather sharp phase and amplitude response. When the synchrotron tune changes, this filter frequency needs to track it. In CESR, the filter frequency is stored as part of an RF save set, although occasional field regulation problems have been known to result in changes in synchrotron tune with current.

The error signal for each bunch is applied to a mixer used to amplitude modulate the $1142 \mathrm{MHz}$ carrier. The electron and positron error signals are summed together and applied to the IF port of the mixer. Phase switching between electrons and positrons is accomplished by switching the carrier signal through two different cable paths to the mixer. Overall phase of the $1142 \mathrm{MHz}$ local oscillator is adjusted using the CESR fast timing system. Resolution is $42 \mathrm{~ns} / 4096 \sim 10.25$ picoseconds or 4.2 degrees at $1142 \mathrm{MHz}$.

The amplitude-modulated carrier signal is applied to a 200 watt TWT amplifier. The amplified signal is equally split three ways and applied to the three drive ports of the kicker cavity. The other three cavity ports are terminated in water-cooled loads. Beam-induced power is substantial. At 1 ampere, the power coupled to each port is expected to be $1 \mathrm{~kW}$. The wideband 1:3 power divider 
couldsuccessfully combine much of this signal and up to 3 $\mathrm{kW}$ could be transmitted to the TWT output.

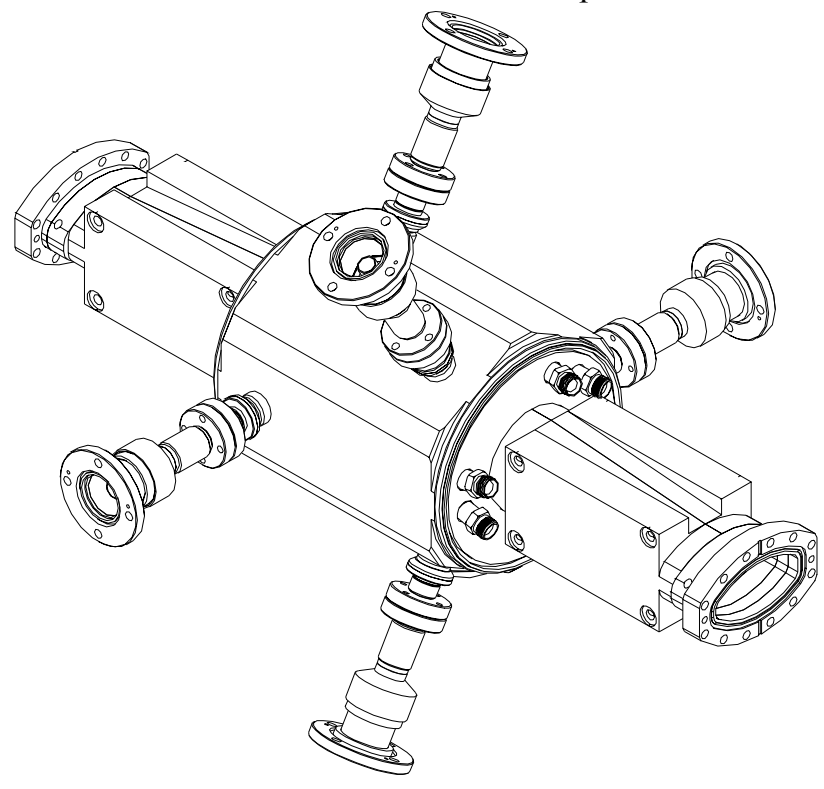

Figure 2: Sketch of the CESR feedback cavity

Table 1: Cavity properties

\begin{tabular}{|c|c|}
\hline Resonance frequency, $f$ & $1142.32 \mathrm{MHz}$ \\
\hline$R / Q\left(R=V^{2} / 2 P\right)$ & $58.1 \mathrm{Ohm}$ \\
\hline$Q_{0}$ (unloaded) & 13,000 \\
\hline$Q_{\mathrm{L}}$ (seen by the RF amplifier) & 27.8 \\
\hline$Q_{\mathrm{L}}$ (seen by the beam) & 13.9 \\
\hline Field decay time, $\tau$ & $3.9 \mathrm{nsec}$ \\
\hline Loss factor (fundamental), $k$ & $0.197 \mathrm{~V} / \mathrm{pC}$ \\
\hline $\begin{array}{c}\text { Power dissipating in a RF load } \\
\text { (9x5 bunches, 0.5 A + 0.5 A) }\end{array}$ & $1.0 \mathrm{~kW}$ \\
\hline $\begin{array}{c}\text { Beam induced voltage } \\
\text { (9x5 bunches, 0.5 A + 0.5 A) }\end{array}$ & $12 \mathrm{kV}$ \\
\hline $\begin{array}{c}\text { Feedback voltage available, } V_{\mathrm{fb}} \\
\text { (with a 200 W TWT amplifier) }\end{array}$ & $630 \mathrm{~V}$ \\
\hline
\end{tabular}

Isolation of the TWT amplifier from the cavity's beaminduced power is needed for two reasons, in addition to the obvious problem of overheating. One is that excess RF voltage could damage the TWT output window. Another potential problem is caused by the fact that the TWT can amplify backwards as well as forward. An in band signal applied to the output is transmitted through the TWT, reflected by whatever mismatch exists at the input and receives full gain on its way back through in the forward direction. This type of crosstalk could substantially reduce the effectiveness of the feedback and it could damage the TWT.

The CESR implementation conservatively isolates the TWT from beam-induced power. In the normal transmission path, there are two circulators at the TWT amplifier output followed by the 1:3 power divider with a circulator and an absorptive lowpass filter in each leg of the power divider output. The lowpass filters attenuate beam-induced power that is out of band and the circulators provide isolation from in band beam-induced power.

Cavity measurements show that higher order modes are well damped by the external loads. However, two modes with high $Q$ values were found: a dipole mode $(f=$ $1726 \mathrm{MHz}, Q=800, R_{\perp}=150 \mathrm{Ohm}$ ) and a monopole mode $\left(f=3220 \mathrm{MHz}, Q=4200, R_{\|}=115 \mathrm{Ohm}\right)$. Fortunately, these have little effect on beam dynamics .

\section{DIAGNOSTICS}

The pre-filter and post-filter data in each DSP are multiplexed into a diagnostic buffer that can be triggered externally. In practice, the feedback system generates its own diagnostic trigger which is also the trigger for the turn-by-turn diagnostic sampling system called COMET.

Figure 3 shows the result of a grow/damp experiment in which the feedback gain is reversed to allow the most unstable modes to grow in amplitude. The feedback gain reverts to the normal sign when the amplitude reaches a programmable trigger level. The ability to reset the feedback gain back to normal using signal amplitude is necessary since the time it takes for the beam amplitude to start growing is not fixed [5]. Damping due to feedback is the difference of the growth and damping rates.

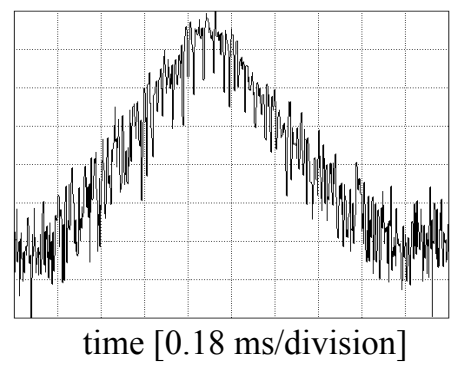

Figure 3: Log RMS amplitude plot of bunch motion during a grow/damp experiment.

\section{REFERENCES}

[1] J.T. Rogers, "Photoemission Instabilities: Theory and Experiment", Particle Accelerator Conference (1997).

[2] J.P. Sikora et al, "Longitudinal Feedback at CESR", Particle Accelerator Conference (1999).

[3] S. Henderson et al, "Improvements to the CESR Injector and Injection Process", these proceedings.

[4] Boni, R. et al. "Kickers and Power Amplifiers for the DA $\Phi N E$ Bunch-by-Bunch Longitudinal Feedback System". Proc. of the Seventh European Part. Accel. Conf. (Vienna, 2000).

[5] J. Fox et al. "Operation and Performance of the PEP II Prototype Longitudinal Damping System at the ALS". Particle Accelerator Conference (1995).

[6] S. Henderson, "A Beamloss Diagnostic System for CESR". Particle Accelerator Conference (1997). 